\title{
ROUND-ROBIN TEST OF SBF FOR IN VITRO MEASUREMENT OF APATITE-FORMING ABILITY OF SYNTHETIC MATERIALS
}

\author{
Hiroaki Takadama ${ }^{1 *}$, Masami Hashimoto ${ }^{1}$, Mineo Mizuno ${ }^{1}$, \\ Tadashi Kokubo ${ }^{2}$ \\ (*Corresponding author: takadama@jfcc.or.jp) \\ ${ }^{1}$ Materials Research and Development Laboratory, Japan Fine Ceramics Center, \\ 2-4-1 Mutsuno, Atsuta-ku, Nagoya 456-8587, Japan \\ ${ }^{2}$ Research Institute for Science and Technology, Chubu University, \\ 1200 Matsumoto-cho, Kasugai, Aichi 487-8501, Japan
}

\begin{abstract}
A number of researchers have used a simulated body fluid (SBF) to get an in vitro preliminary assessment of bone-bonding ability of an artificial material by examining apatite-forming ability in the SBF, before animal experiments. However, the common method for preparing the SBF and for detecting an apatite formed on a surface of a material in the SBF has not been established yet. The ion concentrations of the conventional SBF are not exactly equal to those of human blood plasma. In the present study, the detailed preparing method, and a newly improved SBF were proposed. In vitro assessments of apatite-forming ability in the conventional and proposed SBFs were performed.
\end{abstract}

(Received June 23, 2004; Accepted July 30, 2004)

\section{INTRODUCTION}

It has been revealed that various kinds of materials bond to living bone through a layer of apatite formed on their surfaces in the living body.$^{1-3}$ It has been also shown that this apatite layer can be reproduced on their surfaces even in an acellular and protein-free simulated body fluid (SBF) with ion concentrations nearly equal to those of human blood plasma, and that apatite thus formed is very similar to the bone mineral in its composition and structure. ${ }^{4-14}$ On the basis of these results, a number of researchers have evaluated in vivo bone-bonding ability of a material by examining in vitro apatite-forming ability on its surface in the SBF preliminary to animal experiments. It is quite useful to save the lives of test animals, and reduce the experimental period and cost.

It is, however, known that the ion concentrations of conventional SBF (c-SBF) are not exactly equal to those of human blood plasma. Recently, several revised SBFs such as revised SBF (r-SBF) and modified SBF (m-SBF) with ion concentrations equal to and close to those of human blood plasma, respectively, have been proposed. ${ }^{15,16}$ However, the r-SBF and m-SBF with high $\mathrm{HCO}_{3}{ }^{-}$concentration tend to deposit calcite in addition to bonelike apatite especially on Ca-based bioactive materials due to the release of $\mathrm{Ca}^{2+}$ ion. Therefore, the r-SBF and $\mathrm{m}-\mathrm{SBF}$ are not the best media for bioactivity assessment.

It is also known that the assessment using SBF does not always show good reproducibility. It usually depends on misunderstanding or the lack of care for preparing method of the SBF, as its detailed preparing method has not been established yet. Another possible reason lies in the conventional preparing process, i.e., powder dissolving process. It is considered that the radical increase in the local supersaturation with respect to apatite or calcite raised by dissolving powder reagents can lead to the undesirable precipitation of apatite or calcite during the preparation process. Therefore, it is recently proposed that liquid mixing processes is better to reduce the risk of undesirable precipitation.

On the basis of above opinions and previous works, the composition and the preparation procedure were modified and a newly improved SBF (n-SBF) with ion concentrations equal to those of human blood plasma excepting that of $\mathrm{HCO}_{3}{ }^{-}$was proposed. ${ }^{17}$ The n-SBFs were prepared by two different preparing processes of powder dissolving and liquid mixing processes, respectively. The detailed procedures for preparing the SBFs were also specified. The comparison of in vitro measurement of apatite-forming ability was carried out between the c-SBF and n-SBFs

The result of round robin tests (RRT) of the SBFs for in vitro measurements of apatite-forming ability are reported in the present paper. Ten institutes, including universities, companies and public institutes, took part in this RRT, as shown in Table 1. The authors organized and summarized this RRT under the supervision of Prof. Kokubo, who is the inventor of the $\mathrm{SBF}^{4}$ On the basis of the results of the RRT, instructions to prepare stable SBFs with good reproducibility is provided. 
Table 1. The type, institutes and principal investigator of RRT

\begin{tabular}{ll}
\hline \multicolumn{1}{c}{ Institutes } & Principal investigator \\
\hline Tokyo Medical and Dental University & K. Yamashita \\
Tokyo Dental College & M. Yoshinari \\
Tokyo Institute of Technology & Y. Ohba \\
Nagoya Institute of Technology & T. Kasuga \\
Nara Institute of Science and Technology & C. Ohtsuki \\
Okayama University & A. Osaka \\
Kobe Steel, Ltd. & J. Suzuki \\
Mitsubishi Materials Corporation & N. Asaoka \\
National Institute of Advanced Industrial Science and Technology & Y. Yokogawa \\
Japan Fine Ceramics Center & M. Mizuno \\
\hline
\end{tabular}

\section{MATERIALS AND METHODS}

The c-SBF and two kinds of proposed n-SBFs were prepared following the detailed instructions. The ion concentrations of them are shown in Table 2. The reagents, purity and quantities for the preparation of $1000 \mathrm{~mL}$ of the SBFs are shown in Table 3. All reagents for the preparation of the SBFs were supplied by Nacalai Tesque, Inc., Japan.

The c-SBF is abbreviated from conventional SBF. ${ }^{4}$ It has ion concentrations equal to those of human blood plasma excepting that of $\mathrm{HCO}_{3}^{-}$and $\mathrm{Cl}^{-}$. The concentration of $\mathrm{HCO}_{3}^{-}$is lower and $\mathrm{Cl}^{-}$is higher than that of human blood plasma. It was prepared by dissolving the reagents shown in Table 3 into the solution one by one, i.e., powder dissolving process. It was buffered by tris-hydroxymethyl aminomethane (TRIS) and $1.0-\mathrm{M} \mathrm{HCl}$ at $\mathrm{pH} 7.40$ at $36.5^{\circ} \mathrm{C}$. In the process of $\mathrm{pH}$ adjustment, TRIS was dissolved little by little into the solution to increase the $\mathrm{pH}$ gradually up to 7.45 , and then $1 \mathrm{M}-\mathrm{HCl}$ and TRIS were added alternately, keeping the $\mathrm{pH}$ within the range of 7.42 to 7.45 , until the whole amount of TRIS was dissolved. Then, $\mathrm{pH}$ was adjusted at 7.40 exactly at $36.5{ }^{\circ} \mathrm{C}$ finally.

The np-SBF is abbreviated from n-SBF prepared by powder dissolving process in the similar manner to the c-SBF. It is designed to have concentrations of ions equal to those of human blood plasma excepting that of $\mathrm{HCO}_{3}^{-}$, the concentration of which is set at the same as that of the c-SBF. It was buffered by
2-(4-(2-Hydroxyethyl)-1-piperazinyl)ethane sulfonic acid (HEPES) and $1.0-\mathrm{M} \mathrm{NaOH}$ at pH 7.40 at $36.5^{\circ} \mathrm{C}$. The dissolving order of reagents was modified. The HEPES and $\mathrm{NaOH}$ are dissolved first of all to keep the $\mathrm{pH}$ constant and $\mathrm{NaHCO}_{3}$ was dissolved just before the $\mathrm{pH}$ adjustment as shown in Table 3 to prevent the volatilization of carbon dioxide $\left(\mathrm{CO}_{2}\right)$.

The nl-SBF is abbreviated from n-SBF prepared by liquid mixing process. It has almost same ion concentrations as np-SBF. It was prepared by mixing the $\mathrm{Ca}$ and $\mathrm{P}$ solutions prepared separately in advance. As shown in Table 2, the Ca solution was designed to have twice higher $\mathrm{Ca}^{2+}$ concentration than that of human blood plasma but not to contain $\mathrm{HPO}_{4}{ }^{2-}$ and $\mathrm{HCO}_{3}{ }^{-}$to prevent precipitations of calcium phosphate and calcite, while the concentrations of the other ions and $\mathrm{pH}$ were designed to be equal to those of human blood plasma. The $\mathrm{P}$ solution was designed to have twice higher $\mathrm{HPO}_{4}{ }^{2-}$ and $\mathrm{HCO}_{3}^{-}$concentrations than those of human blood plasma but not to contain $\mathrm{Ca}^{2+}$, while the concentrations of the other ions and $\mathrm{pH}$ were designed to be equal to those of human blood plasma. This liquid mixing procedure can prevent the local supersaturation with respect to apatite or calcite from increasing radically and reduce the risk of undesirable precipitation during the preparation process.

Ion concentrations of the SBFs were measured by inductively coupled plasma (ICP) emission spectroscopy (IRIS Advantage, Nippon Jarrell-Ash. Co. Japan) and ion chromatograph (DX-100, Dionex Corporation, USA). The $\mathrm{pH}$ of SBFs were measured by a pH meter (Mettler-Toledo MA-235, Germany).

Table 2. Nominal ion concentrations of the SBFs in comparison with those of human blood plasma and Ca and $\mathrm{P}$ solutions for preparing the $\mathrm{nl}-\mathrm{SBF}$

\begin{tabular}{|c|c|c|c|c|c|}
\hline \multirow{3}{*}{ Ion } & \multicolumn{5}{|c|}{ Concentration $/ \mathrm{mM}$} \\
\hline & \multirow{2}{*}{ Blood Plasma } & \multirow{2}{*}{ C-SBF } & \multirow{2}{*}{$n p-S B F$} & \multicolumn{2}{|c|}{ nl-SBF } \\
\hline & & & & Ca solution & $\mathrm{P}$ solution \\
\hline $\mathrm{Na}^{+}$ & 142.0 & 142.0 & 142.0 & 142.0 & 142.0 \\
\hline $\mathrm{K}^{+}$ & 5.0 & 5.0 & 5.0 & 5.0 & 5.0 \\
\hline $\mathrm{Mg}^{2+}$ & 1.5 & 1.5 & 1.5 & 1.5 & 1.5 \\
\hline $\mathrm{Ca}^{2+}$ & 2.5 & 2.5 & 2.5 & 5.0 & 0 \\
\hline $\mathrm{Cl}^{-}$ & 103.0 & 147.8 & 103.0 & 103.0 & 103.0 \\
\hline $\mathrm{HCO}_{3}^{-}$ & 27.0 & 4.2 & 4.2 & 0 & 8.4 \\
\hline $\mathrm{HPO}_{4}{ }^{2-}$ & 1.0 & 1.0 & 1.0 & 0 & 2.0 \\
\hline $\mathrm{SO}_{4}{ }^{2-}$ & 0.5 & 0.5 & 0.5 & 0.5 & 0.5 \\
\hline
\end{tabular}


Round-Robin Test of SBF for In Vitro Measurement of Apatite-Forming Ability of Synthetic Materials

Table 3. Reagents, their purities and amounts for preparing $1000 \mathrm{~mL}$ of the SBFs

\begin{tabular}{|c|c|c|c|c|c|}
\hline \multirow{3}{*}{ Reagent* } & \multirow{3}{*}{ Purity $/ \%$} & \multicolumn{4}{|c|}{ Amount'g } \\
\hline & & \multirow[b]{2}{*}{ C-SBF } & \multicolumn{3}{|c|}{ n-SBF } \\
\hline & & & Total & $\begin{array}{c}\text { Ca solution } \\
(500 \mathrm{~mL})\end{array}$ & $\begin{array}{l}\text { P solution } \\
(500 \mathrm{~mL})\end{array}$ \\
\hline $1.0 \mathrm{M}-\mathrm{NaOH}$ & -- & $\cdots$ & $40 \mathrm{~mL}$ & $20 \mathrm{~mL}$ & $20 \mathrm{~mL}$ \\
\hline HEPES** & $>99.0$ & -2 & 21.183 & 10.591 & 10.591 \\
\hline $\mathrm{NaCl}$ & $>99.5$ & 8.035 & 5.404 & 2.702 & 2.702 \\
\hline $\mathrm{NaHCO}_{3}$ & $>99.5$ & 0.355 & -2 & -- & -- \\
\hline $\mathrm{KCl}$ & $>99.5$ & 0.225 & 0.225 & 0.112 & 0.112 \\
\hline $\mathrm{K}_{2} \mathrm{HPO}_{4} \cdot 3 \mathrm{H}_{2} \mathrm{O}$ & $>99.0$ & 0.231 & 0.231 & --- & 0.231 \\
\hline $\mathrm{MgCl}_{2} \cdot 3 \mathrm{H}_{2} \mathrm{O}$ & $>98.0$ & 0.311 & 0.311 & 0.156 & 0.156 \\
\hline 1.0M-HCl & -- & $39 \mathrm{~mL}$ & -4 & --- & --- \\
\hline $\mathrm{CaCl}_{2}$ & $>95.0$ & 0.292 & 0.292 & 0.292 & --- \\
\hline $\mathrm{Na}_{2} \mathrm{SO}_{4}$ & $>99.0$ & 0.072 & 0.072 & 0.036 & 0.036 \\
\hline $\mathrm{NaHCO}_{3}$ & $>99.5$ & 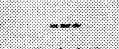 & 0.355 & --- & 0.355 \\
\hline TRIS*** & $>99.0$ & 6.118 & - & --- & --- \\
\hline 1.0M-HCl & -- & $.0 .2 \mathrm{ml}$ & $-\cdots$ & -- & -- \\
\hline $1.0 \mathrm{M}-\mathrm{NaOH}$ & -- & & $-4.8 \mathrm{~mL}$ & $\sim 2 \mathrm{~mL}$ & $\sim 3 \mathrm{~mL}$ \\
\hline
\end{tabular}

*Listed in sequence of dissolution.

**HEPES: 2-(4-(2-Hydroxyethyl)-1- piperazinyl) ethane sulfonic acid.

***TRIS: Tris-hydroxymethylaminomethane.

Table 4. The compositions of the $\mathrm{SiO}_{2}-\mathrm{Na}_{2} \mathrm{O}-\mathrm{CaO}$ glasses

\begin{tabular}{cccc}
\hline \multirow{2}{*}{ Glass } & \multicolumn{3}{c}{ Composition $/ \mathrm{mol} \%$} \\
\cline { 2 - 4 } & $\mathrm{SiO}_{2}$ & $\mathrm{Na}_{2} \mathrm{O}$ & $\mathrm{CaO}$ \\
\hline Glass A & 50 & 25 & 25 \\
Glass B & 55 & 22.5 & 22.5 \\
\hline
\end{tabular}

Two bioactive $\mathrm{CaO}-\mathrm{SiO}_{2}-\mathrm{Na}_{2} \mathrm{O}$ glasses named glass $A$ and $B$ were used to assess the c-SBF, np-SBF and nl-SBF. It has been reported that these glasses show a correlation between bone-forming ability of materials implanted into a bone defect and apatite-forming ability on its surface in the SBF. ${ }^{14}$ Their compositions are shown in Table 4 . The glasses with mirror or \#400-abraded surface were prepared for each glass. One piece of the glass A was immersed in $20 \mathrm{~mL}$ of each SBF at $36.5^{\circ} \mathrm{C}$ for 6,12 and $18 \mathrm{~h}$ and glass B for $12,18,24$, and $30 \mathrm{~h}$, respectively. After removal from each SBF, specimens were washed with distilled water and dried in air. The surfaces of specimens were examined by thin-film X-ray diffraction (TF-XRD: Model-265A, Rigaku Corporation, Osaka, Japan) and scanning electron microscope (FE-SEM: JSM-6330F, JEOL DATUM Co. Ltd., Nagoya, Japan).

\section{RESULTS}

It goes without saying that the c-SBF was prepared successfully with taking great care of $\mathrm{pH}$ change. The $\mathrm{np}-\mathrm{SBF}$ and nl-SBF were prepared successfully by the newly proposed procedure as well as c-SBF. However, it took almost twice more time and labor to prepare the nl-SBF than the c-SBF and np-SBF.

Figure 1 shows the changes in $\mathrm{pH}$ of the solutions in the preparation process of the c-SBF and np-SBF. The $\mathrm{pH}$ became quite low after adding aqueous
$1.0 \mathrm{M}-\mathrm{HCl}$ and the large $\mathrm{pH}$ change was involved in the preparing process of the c-SBF. On the other hand, the $\mathrm{pH}$ was relatively constant and the small $\mathrm{pH}$ change was involved in the preparation process of the $n p-S B F$, because the buffer was added from the beginning. In the preparation process of the nl-SBF, both the $\mathrm{Ca}$ and $\mathrm{P}$ solutions were prepared showing the quite similar $\mathrm{pH}$ changes to the $\mathrm{np}-\mathrm{SBF}$. The $\mathrm{pH}$ kept 7.40 even after mixing $\mathrm{Ca}$ and $\mathrm{P}$ solutions to prepare the nl-SBF.

All the as-prepared SBFs were transparent without any precipitation. Figure 2 shows ion concentrations and $\mathrm{pH}$ of the SBFs as-prepared and after stored for 4 weeks, in comparison with those of human blood plasma. The as-prepared c-SBF showed ion concentrations almost equal to nominal values listed in Table 2, excepting those of $\mathrm{Cl}^{-}$and $\mathrm{HCO}_{3}^{-}$. The measured $\mathrm{Cl}^{-}$concentration was higher and $\mathrm{HCO}_{3}^{-}$ was lower than the nominal values. On the other hand, the as-prepared np-SBF and nl-SBF showed ion concentrations almost equal to nominal values, including those of $\mathrm{Cl}^{-}$and $\mathrm{HCO}_{3}^{-}$. There was little difference in ion concentrations between the np-SBF and nl-SBF, since the nominal compositions were same. The c-SBF and both n-SBFs kept their ion concentrations and $\mathrm{pH}$ unchanged over 4 weeks at $36.5^{\circ} \mathrm{C}$. They kept clear and transparent even after the storage for 8 weeks at $36.5^{\circ} \mathrm{C}$.

Figure 3 shows the XRD patterns of the surfaces of glass $A$ and $B$ with mirror or \#400-abraded surfaces 
Takadama H, Hashimoto M, Mizuno M, Kokubo T after soaked in the c-SBF, np-SBF and nl-SBF for various periods, respectively. Regardless of SBF, the glass $\mathrm{A}$ and $\mathrm{B}$ showed the first XDR patterns of apatite after soaking of $12 \mathrm{~h}$ and $18 \mathrm{~h}$, respectively. Both of the first detection time of apatite formed on glass $A$ and $B$ was less affected by the difference in the surface finishes.
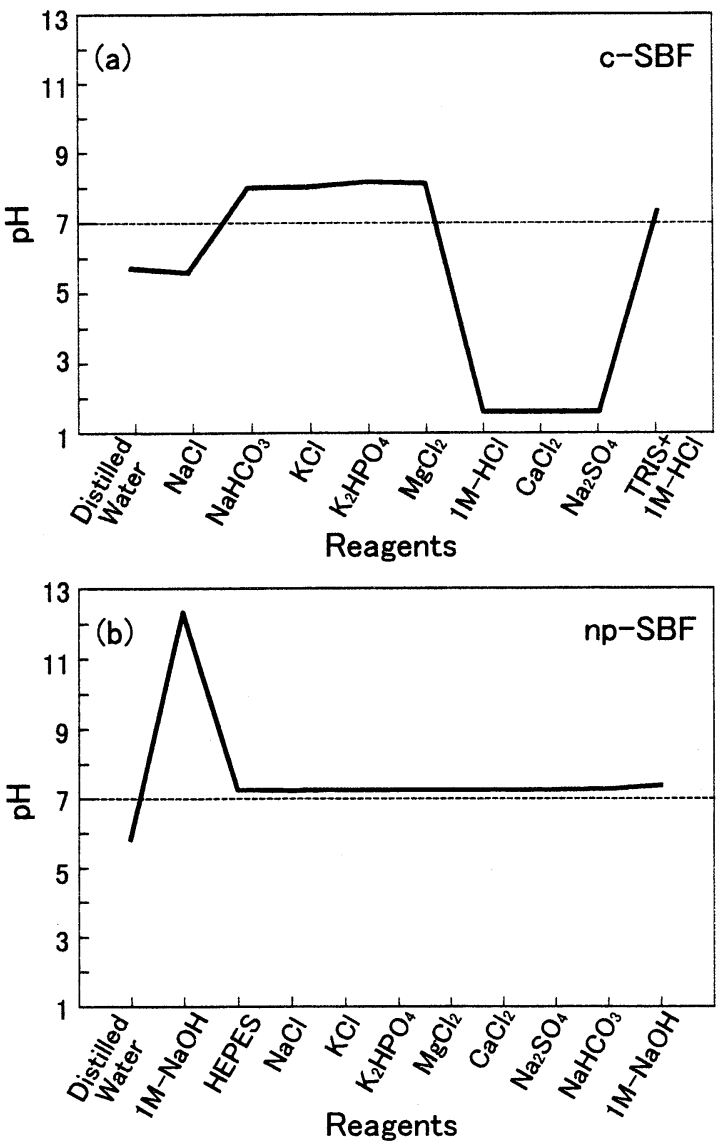

Figure 1. The $\mathrm{pH}$ changes of the solutions in the preparation processes of (a)c-SBF and (b)np-SBF.
Figure 4 shows the SEM images of the surfaces of the glass $A$ and $B$ with mirror and $\# 400$-abraded surfaces after soaked in the c-SBF, np-SBF and nl-SBF for 12 and $18 \mathrm{~h}$, respectively. Regardless of SBF, both glass A and B formed apatite uniformly on the whole surfaces of them. However, the apatite formed on the \#400-abraded glass surfaces had more cracks and bonded more tightly to the glass specimens than that on the mirror surface glasses.

Figure 5 shows the results of the RRT of in vitro assessments of the apatite-forming ability of glass A and $B$ in various SBFs. In total, 9 results of XRD and 11 results of SEM were gathered. The glass $A$ soaked in the c-SBF, np-SBF or nl-SBF showed the quite similar XRD and SEM results, respectively. The glass B soaked in different SBFs also showed the similar results as the glass $A$. The induction periods to form apatite on the surfaces of the glass A and B, respectively, did not depend on the types of SBFs. While the c-SBF showed the relatively high reproducibility, the np-SBF and nl-SBF showed slightly higher reproducibility than $\mathrm{c}-\mathrm{SBF}$, and there were no great differences between the np-SBF and nl-SBF. The SEM analyses tended to detect the apatite formation earlier than XRD. The SEM analyses showed smaller data spread than XRD. The intensity of XRD patterns differed from one institute to another, and some institutes did not showed any XRD patterns. It was found in almost every glass that some portions of apatite layer formed on the glasses were peeled off. The area of the peel-off also differed from one institute to another, and the glass with $\# 400$-abraded surface showed smaller area of the peel-off in total than that with mirror surface. It became quite difficult to get SEM images of the glass surfaces with the peel-off, as it induced the intense charge up.

As for the preparation of the nl-SBF, a lot of comments were received that the nl-SBF is not easy-to-prepared, as it took almost twice more time and labor to prepare nl-SBF than the c-SBF and np-SBF.
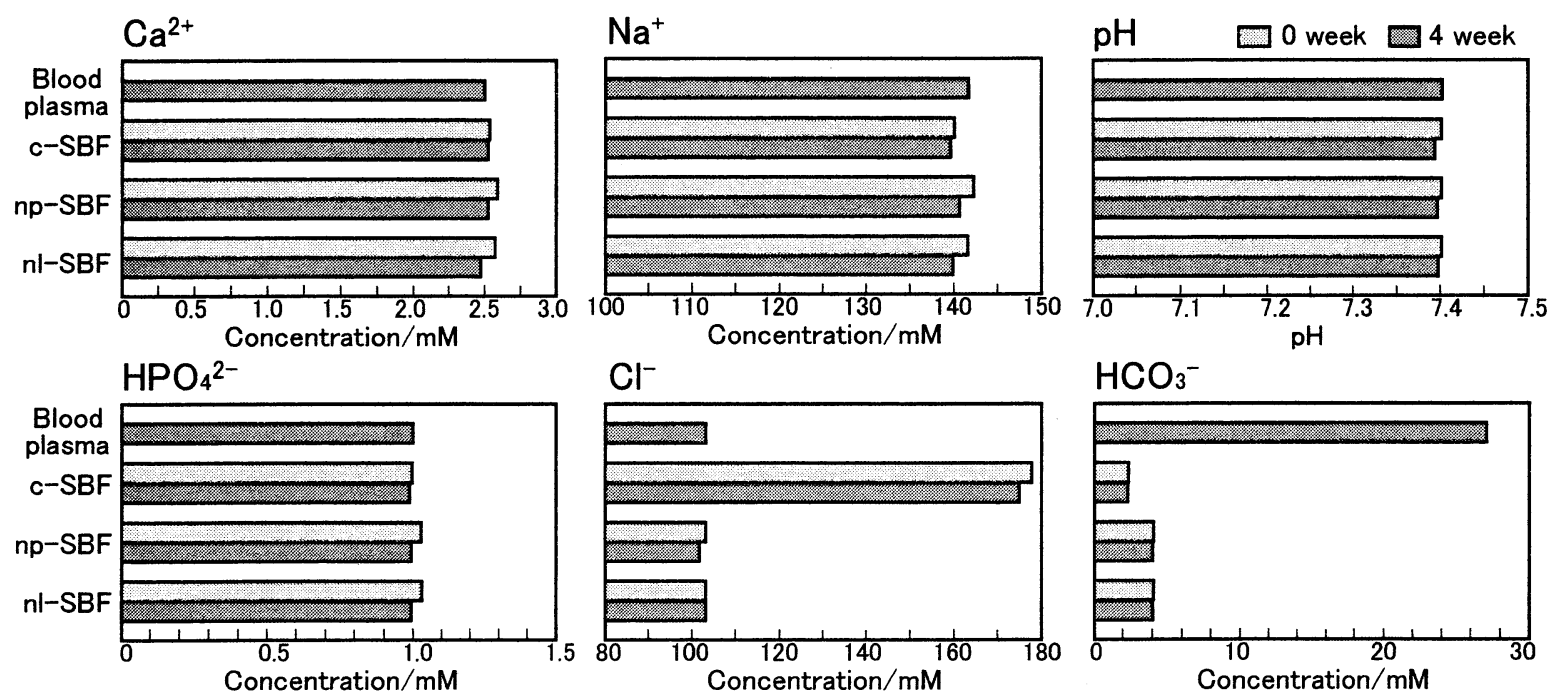

Figure 2. The ion concentrations and $\mathrm{pH}$ of the c-SBF, np-SBF and nl-SBF as-prepared and after stored for 4 weeks, in comparison with those of human blood plasma. 

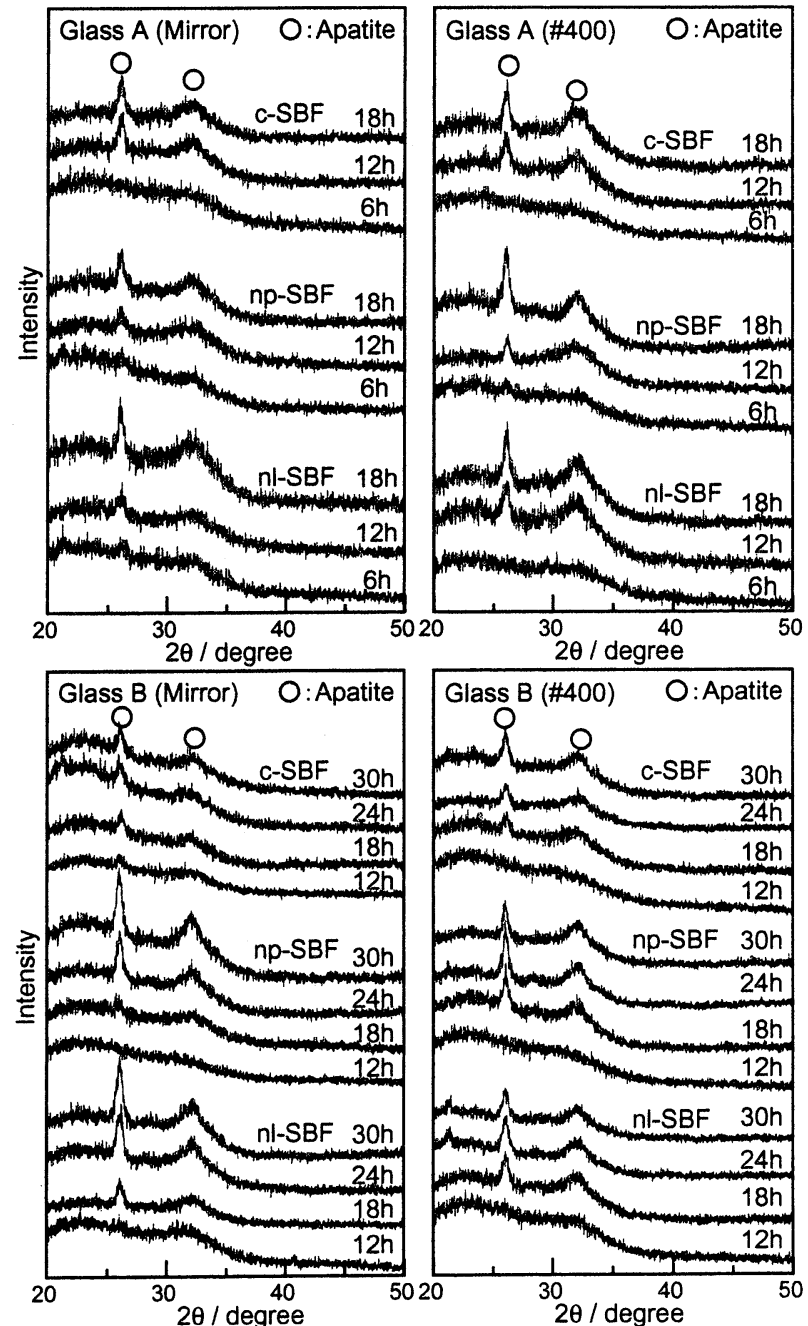

Figure 3. X-ray diffraction patterns of the surfaces of glass A and B with mirror (left) and \#400-abraded surface (right) after soaked in c-SBF, np-SBF and nlSBF for various periods.

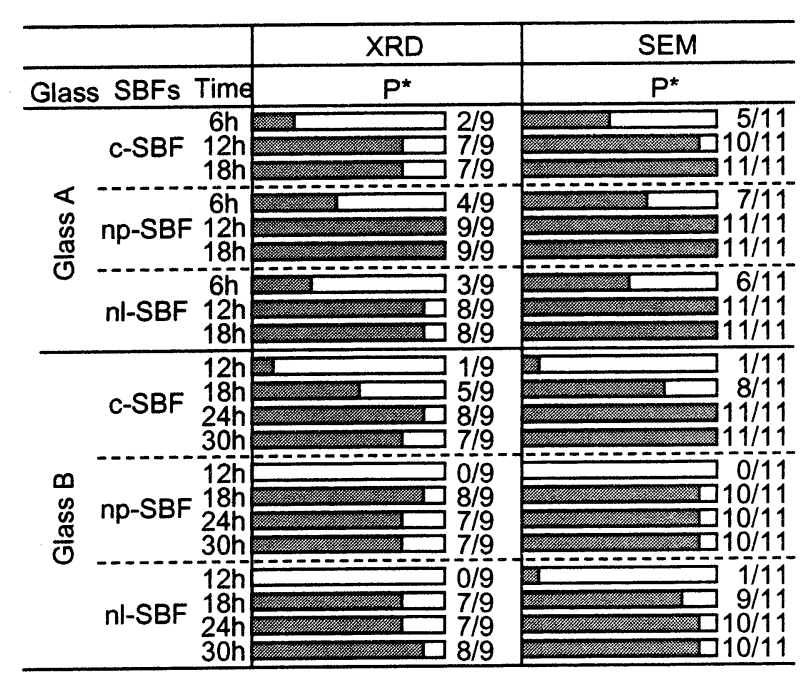

$P^{\star}:$ The rate of institutes which detect the apatite by XRD and SEM (The number of institutes which observed the apatite formation / Total institutes that took part in the RRT.)

Figure 5. Results of SBF round robin tests
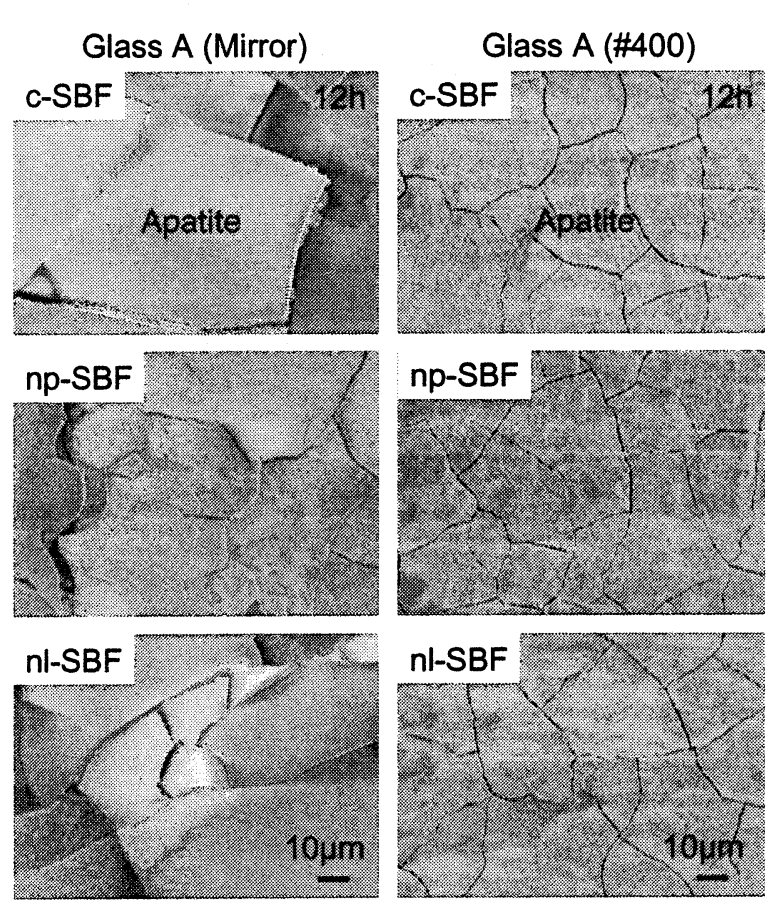

Glass B (Mirror)

Glass B (\#400)
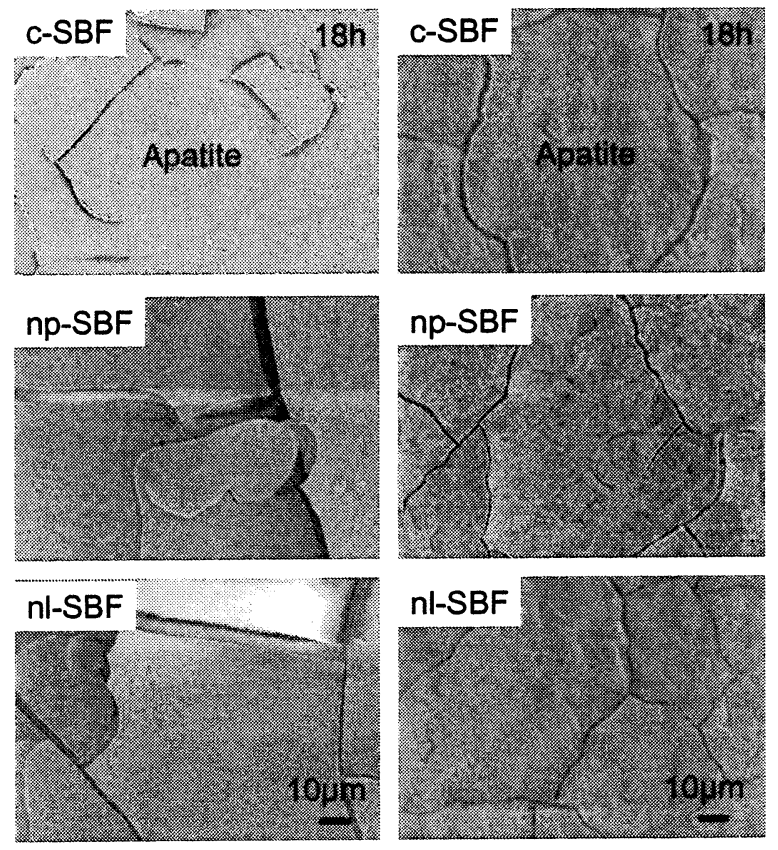

Figure 4. SEM images of the surfaces of the glass A and B with mirror (left) and $\# 400$-abraded surfaces (right) after soaked in c-SBF, np-SBF and nl-SBF for 12 and $18 \mathrm{~h}$.

\section{DISCUSSION}

Generally, the SBFs such as c-SBF and n-SBFs are metastable solutions supersaturated with respect to apatite. An inappropriate preparation method can lead to the homogeneous nucleation of apatite in the solution. However, the detailed instructions for 
Takadama H, Hashimoto M, Mizuno M, Kokubo T

preparing the SBFs are not always well known all over the world. Sometimes, unstable SBFs were prepared by lack in care of instructions. Especially in the preparation of the c-SBF, the time to prepare it and the dissolution processes of $\mathrm{CaCl}_{2}$ and TRIS are quite important. To prepare the c-SBF, the $\mathrm{pH}$ is once lowered by the addition of $1.0 \mathrm{M}-\mathrm{HCl}$ as shown in Figure 1. This can prevent the undesirable precipitation of apatite when the $\mathrm{CaCl}_{2}$ is added, but lead to the volatilization of $\mathrm{CO}_{2}$, simultaneously. Therefore, it is necessary to complete the preparation of the c-SBF without interruptions in a given period every time. In addition, if a large amount of $\mathrm{CaCl}_{2}$ or TRIS were added into the solution at one time, the local $\mathrm{Ca}$ concentration or $\mathrm{pH}$ of the solution increased radically to induce undesirable precipitations of calcite or apatite. Therefore, it is necessary to dissolve these reagents into the solution little by little. It is also necessary not to raise the $\mathrm{pH}$ over 7.45 by adding TRIS, since the c-SBF becomes highly supersaturated with respect to the apatite. Remaining TRIS and $1.0 \mathrm{M}-\mathrm{HCl}$ have to be added alternately into the solution keeping the $\mathrm{pH}$ within the range of 7.42 to 7.45 , until the whole amount of TRIS is dissolved. Then, the $\mathrm{pH}$ is finally adjusted to 7.40 exactly at $36.5^{\circ} \mathrm{C}$. If the $\mathrm{c}-\mathrm{SBF}$ is preparing in this manner, stable c-SBF can be prepared.

However, the concentration of $\mathrm{HCO}_{3}^{-}$in the c-SBF is lower and $\mathrm{Cl}^{-}$is higher than nominal concentration. This is because the $\mathrm{Cl}^{-}$concentration derived from 40 $\mathrm{mL}$ of aqueous $1.0 \mathrm{M}-\mathrm{HCl}$ was not included in the nominal value, and that a portion of $\mathrm{HCO}_{3}{ }^{-}$volatilized as $\mathrm{CO}_{2}$ gas when the $\mathrm{pH}$ became quite low after adding $1.0 \mathrm{M}-\mathrm{HCl}$ in the preparing process of the $\mathrm{c}-\mathrm{SBF}$. Therefore, the $\mathrm{np}-\mathrm{SBF}$ was proposed to bring the $\mathrm{Cl}^{-}$concentration equal to that of human blood plasma and $\mathrm{HCO}_{3}^{-}$concentration close to nominal value by changing the buffer. The HEPES buffer and $\mathrm{NaOH}$ are dissolved first of all to keep the $\mathrm{pH}$ constant and $\mathrm{NaHCO}_{3}$ was dissolved just before the $\mathrm{pH}$ adjustment to prevent the volatilization of $\mathrm{CO}_{2}$. Figure 2 proves that the modified preparing process can prevent its volatilization. Therefore, the proposed np-SBF was prepared successfully.

Additionally, some say that the conventional powder dissolving process is fundamentally at risk of the undesirable precipitations, since the radical increases in local $\mathrm{pH}$ or concentrations around the dissolving powders are inevitable. It is difficult to prepare constantly the SBFs with nominal concentrations and good reproducibility of the apatite-forming ability assessment. Therefore, the liquid mixing process was also proposed to prepare the nl-SBF. Figure 2 showed that the proposed nl-SBF was also prepared successfully.

Using the c-SBF and proposed np-SBF and nl-SBF, the apatite-forming ability tests were performed. Since all the glass $A$ and $B$ formed apatite regardless of SBF as shown in Figure 3 and 4, it is reasonable to say that both the np-SBF and nl-SBF can be also used for the apatite-forming ability assessments. The formed apatite tended to bond more tightly to the glass with \#400-abraded surface than that with mirror surface, since the mechanical interlock between the glass and the apatite is created only on the abraded surfaces. Therefore, the \#400-abraded surface is better than mirror surface for the assessments with high reproducibility.

Since the RRT clarified that both glass A and B showed the quite similar results regardless of SBFs as shown in Figure 5, it is proved that both the np-SBF and nl-SBF can be also used for the apatite-forming ability assessments. The reproducibility of the c-SBF was improved and was higher than originally expected, since all institutes taking part in the RRT prepared the c-SBF following the detailed instructions, which was briefly written above. It is considered that the appropriate procedure for preparing the c-SBF can control the volatilization of $\mathrm{CO}_{2}$ to some extent and made the RRT data spread lower. On the other hand, the reproducibility of the np-SBF and nl-SBF was quite similar and almost all the institutes showed the good reproducibility of the n-SBFs in the RRT, since the procedure for preparing the $n-S B F s$ involves little volatilization of $\mathrm{CO}_{2}$ originally. This indicates the SBFs prepared in an appropriate manner following the instructions can show good reproducibility regardless of the preparing procedure.

The first detection time of apatite formation, however, depends on the analyzing apparatus. The SEM can detect a quite small particle of apatite, while the XRD cannot detect until a certain area are covered. The SEM results less affected by the equipment, while the XRD results largely depend on the experimental conditions and device such as type and power of X-ray lamp. Therefore, the SEM tended to detect the apatite formation earlier and showed smaller data spread than XRD. Considering above reasons, it can be said that the apatite-forming ability does not differ by types of SBFs. It is, however, better to use the $\mathrm{n}-\mathrm{SBF}$, if you intend to prepare the apatite, whose composition is close to that of human natural bone in addition to the apatite-forming ability assessments. Consequently, the results of the RRT performed in various institutes concluded that the proposed np-SBF and nl-SBF as well as c-SBF could be used for the apatite-forming ability assessments.

\section{CONCLUSION}

The proposed np-SBF and nl-SBF as well as c-SBF with good reproducibility were prepared successfully. The bioactive $\mathrm{CaO}-\mathrm{SiO}_{2}-\mathrm{Na}_{2} \mathrm{O}$ glasses soaked even in the proposed np-SBF or nl-SBF as well as c-SBF showed quite similar apatite-forming ability. All SBFs such as c-SBF, np-SBF and nl-SBF can be used for in vitro apatite-forming ability assessments preliminary to animal experiments. 
Round-Robin Test of SBF for In Vitro Measurement of Apatite-Forming Ability of Synthetic Materials

\section{ACKNOWLEDGEMENT}

The authors would like to express great appreciation to Dr. K. Yamashita, Dr. M. Yoshinari, Dr. S. Takemoto, Dr. Y. Ohba, Dr. T. Kasuga, Dr. C. Ohtsuki, Dr. A. Osaka, Dr. J. Suzuki, Dr. N. Asaoka, Dr. Y. Yokogawa and their colleagues for cooperating and participating in the RRT of the SBFs and all of the researchers for providing many opinions about the SBFs.

We also thank to Dr. T. Miyazaki (Kyushu Institute of Technology), Dr. M. Uchida, Dr. A. Oyane (National Institute of Advanced Industrial Science and Technology), Dr. M. Kamitakahara (Nara Institute of Science and Technology), Dr. H.-M. Kim (Yonsei University) and Dr. M. Kawashita (Kyoto University) for cooperating to prepare the detailed instructions for preparing SBFs.

\section{REFERENCES}

1. M. Neo, S. Kotani, T. Nakamura, T. Yamamuro, C. Ohtsuki, T. Kokubo and Y. Bando, J. Biomed. Mater. Res., 26, 1419 (1992).

2. W. Holand, W. Vogel, K. Naumann and J. Gummel, J. Biomed. Mater. Res., 19, 303 (1985).

3. K. Ohura, T. Nakamura, T. Yamamuro, Y. Ebisawa, T. Kokubo, Y. Kotoura, and M. Oka, $J$. Mater. Sci.: Materials in Medicine, 3, 95 (1992).

4. T. Kokubo, H. Kushitani, S. Sakka, T. Kitsugi and T. Yamamuro, J. Biomed. Mater. Res., 24, 721 (1990).

5. S.B. Cho, K. Nakanishi, T. Kokubo, N. Soga, C. Ohtsuki, T. Nakamura, T. Kitsugi, and T. Yamamuro, J. Am. Ceram. Soc., 78 [7], 1769 (1995).

6. M.R. Filguerias, G. LaTorre and L.L. Hench, $J$. Biomed. Mater. Res., 27, 445 (1993).

7. T. Kokubo, H. Kushitani, Y. Ebisawa, T. Kitsugi, S. Kotani, K. Oura and T. Yamamuro, in Bioceramics Vol. 1 , edited by $\mathrm{H}$. Oonishi, $\mathrm{H}$. Aoki and K. Sawai, (Ishiyaku EuroAmerica, Tokyo, 1989), pp. 157-162.

8. T. Himeno, H.-M. Kim, H. Kaneko, M. Kawashita, T. Kokubo and T. Nakamura, in Bioceramics Vol. 15, edited by B. Ben-Nissan, D. Sher and W. Walsh, (Trans Tech Publication Ltd., Switzerland, 2002), pp. 457-460.

9. T. Kokubo, S. Ito, Z. T. Huang, T. Hayashi, S. Sakka, T. Kitsugi and T. Yamamuro, J. Biomed. Mater. Res., 24, 331 (1990).

10. C. Ohtsuki, H. Kushitani, T. Kokubo, S. Kotani and T. Yamamuro, J. Biomed. Mater. Res., 25, 1363 (1991).

11. Y. Ebisawa, T. KoKubo, K. Ohura and T. Yamamuro, J. Mat. Sci.: Mater. Med., 1, 239 (1990).

12. M. Neo, S. Kotani, Y. Fujita, T. Nakamura, T. Yamamuro, Y. Bando, C. Ohtsuki and T. Kokubo, J. Biomed. Mater. Res., 26, 255 (1992).
13. H.-M. Kim, F. Miyaji, T. Kokubo, C. Ohtsuki and T. Nakamura, J. Am. Ceram. Soc., 78 [9] 2405 (1995).

14. S. Fujibayashi, M. Neo, H.-M. Kim, T. Kokubo and T. Nakamura, Biomaterials, 24, 1349 (2003).

15. A. Oyane, K. Onuma, A. Ito, H.-M. Kim, T. Kokubo and T. Nakamura, J. Biomed. Mater. Res., 64A [2], 339 (2003).

16. A. Oyane, H.-M. Kim, T. Furuya, T. Kokubo, T. Miyazaki and T. Nakamura, J. Biomed. Mater. Res., 65A [2], 188 (2003).

17. H. Takadama, M. Hashimoto, M. Mizuno K. Ishikawa and T. Kokubo, in Bioceramics Vol. 16, edited by M.A. Barbosa, F.J. Monteiro, R. Correia and B. Leon, (Trans Tech Pub., Switzerland, 2003), pp. 115-118. 